\title{
Genetic and Clinical Heterogeneity in Thirteen New Cases with Aceruloplasminemia. Atypical Anemia as a Clue for an Early Diagnosis
}

\author{
Marc Vila Cuenca ${ }^{1,+}$, Giacomo Marchi ${ }^{2,+}{ }^{+}$, Anna Barqué ${ }^{1}$, Clara Esteban-Jurado ${ }^{1}$, \\ Alessandro Marchetto ${ }^{3}\left(\mathbb{D}\right.$, Alejandro Giorgetti ${ }^{3}$, Viorica Chelban ${ }^{4,5,6}$, Henry Houlden ${ }^{4,5,7}$, \\ Nicholas W Wood ${ }^{4,7}$, Chiara Piubelli ${ }^{8}$, Marina Dorigatti Borges ${ }^{9}$, \\ Dulcinéia Martins de Albuquerque ${ }^{9}$, Kleber Yotsumoto Fertrin ${ }^{9,10}{ }^{\circledR}$, Ester Jové-Buxeda ${ }^{11}$, \\ Jordi Sanchez-Delgado ${ }^{12,13} \mathbb{D}$, Neus Baena-Díez ${ }^{14}$, Birute Burnyte ${ }^{15}$, Algirdas Utkus ${ }^{15}$, \\ Fabiana Busti $^{2}{ }^{(D)}$, Gintaras Kaubrys ${ }^{16}{ }^{D}$, Eda Suku ${ }^{3}$, Kamil Kowalczyk ${ }^{17}$, \\ Bartosz Karaszewski ${ }^{17}$ (D), John B. Porter ${ }^{18}$, Sally Pollard ${ }^{19}$, Perla Eleftheriou ${ }^{18}$, \\ Patricia Bignell 20, Domenico Girelli ${ }^{2, *(1)}$ and Mayka Sanchez 21,22,23,*(D)
}

1 Iron Metabolism: Regulation and Diseases Group, Josep Carreras Leukaemia Research Institute (IJC), Campus Can Ruti, Badalona, 08916 Barcelona, Spain; m.vila.cuenca@gmail.com (M.V.C.); annabarque@gmail.com (A.B.); darth.clara@gmail.com (C.E.-J.)

2 EuroBloodNet Referral Center for Iron Disorders and Gruppo Interdisciplinare Malattie del Ferro, Internal Medicine Unit, Azienda Ospedaliera Universitaria Integrata di Verona, 37134 Verona, Italy; markallbutone@gmail.com (G.M.); fabiana.busti@gmail.com (F.B.)

3 Department of Biotechnology, University of Verona, 37134 Verona, Italy; alessandromarchetto@outlook.it (A.M.); alejandro.giorgetti@univr.it (A.G.); eda.suku@univr.it (E.S.)

4 National Hospital for Neurology and Neurosurgery, London WC1N 3BG, UK; v.chelban@ucl.ac.uk (V.C.); h.houlden@ucl.ac.uk (H.H.); n.wood@ucl.ac.uk (N.W.W.)

5 Department of Neuromuscular Diseases, Institute of Neurology, University College London, London WC1N 3BG, UK

6 Department of Neurology and Neurosurgery, Institute of Emergency Medicine, Toma Ciorbă 1, Chisinau, MD-2052 Chisinau, Republic of Moldova

7 Neurogenetics Laboratory, The National Hospital for Neurology and Neurosurgery, London WC1N 3BG, UK

8 Centre for Tropical Diseases, Ospedale Sacro Cuore - Don Calabria, 37024 Negrar (VR), Italy; chiara.piubelli@univr.it

9 Hematology and Hemotherapy Center-Hemocentro Campinas, University of Campinas-UNICAMP, Campinas 13083-878, Brazil; ma.borges@yahoo.com.br (M.D.B.); dulmal@unicamp.br (D.M.d.A.); kleber@uw.edu (K.Y.F.)

10 Division of Hematology, Department of Medicine, University of Washington, Seattle, WA 98195, USA

11 Internal Medicine Department, Parc Tauli Hospital Universitari, Institut d' Investigació i Innovació Parc Tauli I3PT, Universidad Autonoma de Barcelona, 08208 Sabadell, Spain; ejoveb@tauli.cat

12 Hepatology Unit, Digestive Diseases Department, Parc Tauli Hospital Universitari. Institut d' Investigació i Innovació Parc Tauli I3PT, Universidad Autonoma de Barcelona, 08208 Sabadell, Spain; jsanchezd@tauli.cat

13 Centro de Investigación Biomedica y en red Enfermedades hepáticas y digestivas (CIBERehd), Instituto de Salud Carlos III, 28029 Madrid, Spain

14 Genetic Department, Parc Tauli Hospital Universitari, Institut d' Investigació i Innovació Parc Tauli I3PT, Universidad Autonoma de Barcelona, 08208 Sabadell, Spain; NBaena@tauli.cat

15 Department of Human and Medical Genetics, Institute of Biomedical Sciences, Faculty of Medicine, Vilnius University, LT-08661 Vilnius, Lithuania; birute.burnyte@gmail.com (B.B.); algirdas.utkus@mf.vu.lt (A.U.)

16 Clinic of Neurology and Neurosurgery, Institute of Clinical Medicine, Faculty of Medicine, Vilnius University, 08661 Vilnius, Lithuania; gintaras.kaubrys@santa.lt

17 Department of Adult Neurology, Medical University of Gdańsk, 80-210 Gdańsk, Poland; kkowalczyk@gumed.edu.pl (K.K.); bartkar@gumed.edu.pl (B.K.)

18 Joint Red Cell Unit, Haematology Department, University College London NHS Foundation Trust, Cancer Services, 250 Euston Road, London NW1 2PG, UK; j.porter@ucl.ac.uk (J.B.P.); perla.eleftheriou@nhs.net (P.E.)

19 Consultant Paediatrician, Bradford Royal Infirmary, Duckworthlane, Bradford BD9 6RJ, UK; sally.pollard1@nhs.net 
20 Oxford Regional Genetics Laboratory, Oxford University Hospitals NHS Foundation Trust, The Churchill Hospital, Oxford OX3 7LE, UK; patricia.bignell@ouh.nhs.uk

21 Iron Metabolism: Regulation and Diseases Group, Department of Basic Sciences, Faculty of Medicine and Health Sciences, Universitat Internacional de Catalunya (UIC); Sant Cugat del Valles, 08017 Barcelona, Spain

22 Program of Program of Predictive and Personalized Medicine of Cancer (PMPPC), Institut d 'Investigació Germans Trias i Pujol (IGTP), Campus Can Ruti, Badalona, 08916 Barcelona, Spain

23 BloodGenetics S.L., Esplugues de Llobregat, 08950 Barcelona, Spain

* Correspondence: domenico.girelli@univr.it (D.G.); msanchezfe@uic.es (M.S.)

+ These authors contributed equally to this work.

Received: 12 March 2020; Accepted: 26 March 2020; Published: 30 March 2020

\begin{abstract}
Aceruloplasminemia is a rare autosomal recessive genetic disease characterized by mild microcytic anemia, diabetes, retinopathy, liver disease, and progressive neurological symptoms due to iron accumulation in pancreas, retina, liver, and brain. The disease is caused by mutations in the Ceruloplasmin $(C P)$ gene that produce a strong reduction or absence of ceruloplasmin ferroxidase activity, leading to an impairment of iron metabolism. Most patients described so far are from Japan. Prompt diagnosis and therapy are crucial to prevent neurological complications since, once established, they are usually irreversible. Here, we describe the largest series of non-Japanese patients with aceruloplasminemia published so far, including 13 individuals from 11 families carrying 13 mutations in the $C P$ gene ( 7 missense, 3 frameshifts, and 3 splicing mutations), 10 of which are novel. All missense mutations were studied by computational modeling. Clinical manifestations were heterogeneous, but anemia, often but not necessarily microcytic, was frequently the earliest one. This study confirms the clinical and genetic heterogeneity of aceruloplasminemia, a disease expected to be increasingly diagnosed in the Next-Generation Sequencing (NGS) era. Unexplained anemia with low transferrin saturation and high ferritin levels without inflammation should prompt the suspicion of aceruloplasminemia, which can be easily confirmed by low serum ceruloplasmin levels. Collaborative joint efforts are needed to better understand the pathophysiology of this potentially disabling disease.
\end{abstract}

Keywords: aceruloplasminemia; ceruloplasmin; iron metabolism; neurodegenerative disease; anemia; ferritin

\title{
1. Introduction
}

Aceruloplasminemia (ACP) (OMIM\#604290, ORPHA48818) is an adult-onset rare autosomal recessive disorder due to mutations in the $C P$ gene (3q24-q25) encoding ceruloplasmin $(C P)$, a copper-containing ferroxidase involved in maintaining iron homeostasis $[1,2]$. The pathological basis of ACP includes mild microcytic anemia and iron overload in several tissues, which can lead to diabetes mellitus, liver disease, progressive neurodegeneration, and retinopathy [1]. ACP was first described in 1987 in a 52-year-old Japanese female suffering from retinal degeneration, diabetes mellitus, and blepharospasm [3]. Usually, the onset of clinical manifestations is around the fourth or fifth decade of life, while biochemical signs of mild microcytic anemia with low transferrin saturation (TSAT) and paradoxically high ferritin may be evident since childhood [2]. Its prevalence is estimated at about one per two million offspring in Japanese non-consanguineous marriages, while epidemiologic data in the non-Japanese population are substantially missing [1].

Diagnosis is usually based on the evidence of very low or undetectable levels of serum CP and clinical, biochemical, or radiologic signs of iron overload in target organs. Current treatments are mainly based on iron-chelating agents, which are quite effective in reducing liver iron accumulation and may prevent further brain iron deposition but are poorly effective in patients with already established neurologic damage [4-6]. Administration of fresh-frozen human plasma [7], vitamin E, and oral 
administration of zinc [8] have also been reported. The administration of a CP enzyme replacement therapy showed promising results in CP-knockout mice [9].

Understanding the pathophysiology of ACP is of interest also in other more common neurodegenerative disorders, such as Alzheimer's and Parkinson's diseases, characterized by an alteration of brain iron homeostasis [10]. CP is a single polypeptide chain of 1046 amino acids that can bind up to six atoms of copper. $\mathrm{CP}$ has six structural domains with a three-copper catalytic center, which is crucial for its oxidative function [11] and protein stability [12]. Two isoforms of CP are produced by alternative splicing in exons 19 and 20: a soluble form and a glycosylphosphatidylinositol (GPI)-anchored membrane form [13]. The soluble form is almost exclusively synthesized by hepatocytes, while the GPI-anchored membrane isoform is expressed by a number of cells, including brain astrocytes glial cells, hepatocytes, macrophages, pancreatic, and retinal epithelial cells [14]. This membrane isoform plays a key role in cellular iron egress cooperating with ferroportin, the ubiquitous and unique transmembrane protein able to export iron from the cells $[15,16]$. CP, with its copper-mediated oxidation of ferrous iron $\left(\mathrm{Fe}^{2+}\right)$ to ferric iron $\left(\mathrm{Fe}^{3+}\right)$, ensures the appropriate binding of extracellular iron to transferrin [1]. Since macrophages are key cells in iron recycling, a macrophage iron overload would be expected in ACP. Conversely, iron accumulation is usually observed in hepatocytes/liver (Figure 1) rather than in macrophages/spleen in affected patients, suggesting that additional pathophysiologic roles of CP are probably involved [17]. Specifically, it has been reported that GPI-anchored CP can also modulate the interaction between ferroportin and hepcidin [18-20], the master regulator of systemic iron homeostasis [21]. Furthermore, even if soluble CP is not able to pass the blood-brain barrier, it is also present in the brain through secretion by epithelial cells of the choroid plexus into the cerebrospinal fluid [22], and the role of this soluble form in the brain is still unclear. Overall, it emerges that $\mathrm{CP}$ is part of a complex regulatory system involved in maintaining iron balance, but further research is needed to better elucidate ACP pathophysiology, hopefully helping to develop more efficient treatments.

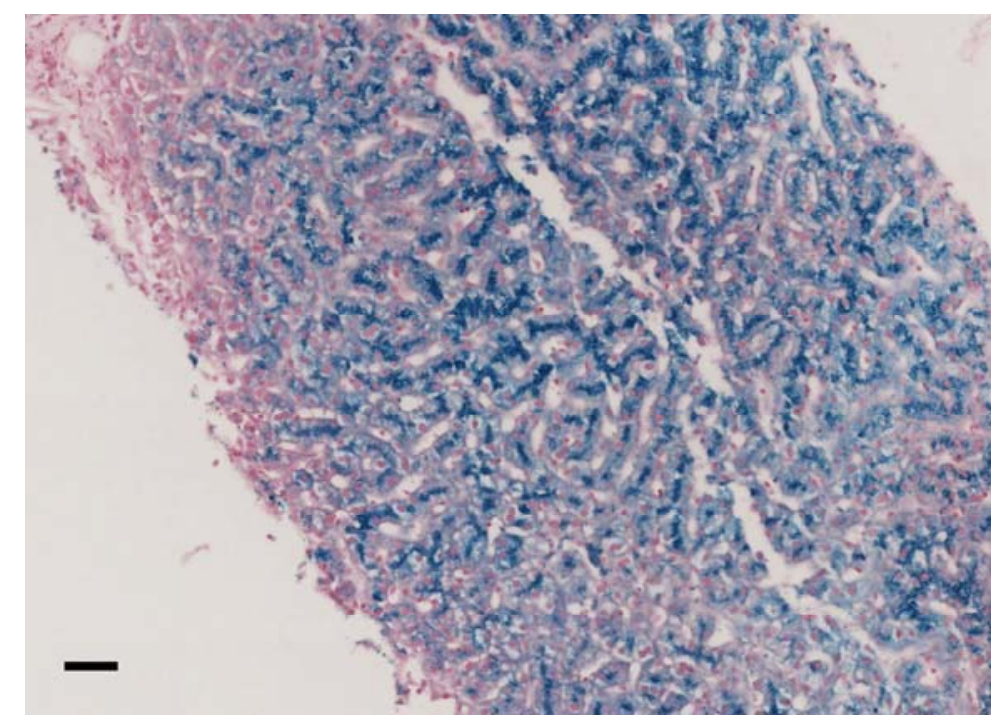

Figure 1. Liver histology of family 7 proband II.2, pearls stain $(20 \times$, scale bar $=100 \mu \mathrm{m})$. Biopsy analysis showed preserved liver architecture with marked iron deposition in hepatocytes, predominating at the biliary pole of the cells.

To address this need, in our international collaborative study, we describe the largest series of non-Japanese patients with ACP published so far, including 13 individuals from 11 families carrying 13 mutations in the $C P$ gene, 10 of which are novel. 


\section{Patients and Methods}

\subsection{Patients}

We got written informed consent from patients and relatives. Project: Uncovering new molecular and pathophysiological networks in iron metabolism (SAF2015-70412-R) approved on 10 July 2015 by the Ethics Research Committee from the Hospital Germans Trias i Pujol, Badalona, Spain. Protocol n.36792 from Project n.1460 was approved by the Ethics Research Committee from Hospital of Verona, Italy. Obtained informed written consent for molecular studies was obtained from all of the patients in accordance with the Declaration of Helsinki 1975, as revised in 2008 for The Diagnostic Lab Oxford Medical Genetics Laboratories from Oxford University Hospitals NHS Foundation Trust, United Kingdom. A detailed description of the biochemical, clinical, and genetic data of the 13 affected members in the 11 studied families with ACP is provided in the Supplementary Methods.

\subsection{DNA Extraction, PCR Amplification, and DNA Sequencing}

Genetic studies were performed with minor differences for all the pedigrees. Genomic DNA was extracted from peripheral blood using the FlexiGene DNA kit (Qiagen) or Wizard Genomic Purification kit (Promega, Madison, WI) or phenol-chloroform extraction (QIAsymphony DNA extraction kits, Qiagen) according to manufacturer's instructions.

Ceruloplasmin gene regions (exonic, intron-exon boundaries, and untranslated regions) were sequenced using the Sanger method or Next-Generation Sequencing (NGS) methods.

For Sanger methods, the CP gene was amplified using $50 \mathrm{ng}$ of genomic DNA. Primer sequences and PCR conditions are available upon request. For patients in families 9 and 10, apart from sequencing the regions of the $C P$ gene in which most mutations have been described [19], the whole CP pseudogene $(C P P)$ was also sequenced. The resulting amplification products were verified on a $2 \%$ ethidium bromide agarose gel. The purified PCR products were sequenced using the conventional Sanger method. Sequencing results were analyzed using Mutation Surveyor software (SoftGenetics LLC, PA, USA) or Chromas software (Technelysium Pty Ltd., Australia).

For NGS methods, a patient in family 2 was analyzed using the targeted NGS gene panel (v14) for iron-related anemias that included the following 5 genes: CP, TF, TMPRSS6, SLC11A2, STEAP3. For patients in families 7 and 8, NGS sequencing was performed by targeted capture and sequencing of $C P$, along with the five canonical "hemochromatosis" genes (HFE, HFE2, HAMP, TFR2, and SLC40A1), as previously described [23]. Patients in families 4, 11, and 12 were analyzed using a targeted NGS gene panel including the following genes: HFE, HJV, HAMP, TFR2, SLC40A1, ALAS2, CP, HEPH, TF, FTH1, FTL, SLC11A2, TMPRSS6, BMP4, BMP6, and SMAD4.

Briefly, the capture of key genomic regions of interest was conducted starting from $225 \mathrm{ng}$ of gDNA using a custom design HaloPlex ${ }^{\mathrm{TM}}$ Target Enrichment 1-500 kb Kit (Agilent Technologies, Santa Clara, CA) or TruSeq ${ }^{\circledR}$ Custom Amplicon version 1.5 kits (Illumina, CA) according to manufacturer instructions. Library quality was determined using the Agilent High Sensitivity DNA kit on the Agilent 2100 bioanalyzer or Agilent 4200 TapeStation System. Libraries were sequenced with the NextSeq 500 High Output Reagent Cartridge v2 (300 cycles) or MiSeq reagent kit v2 (300 cycles) (Illumina, San Diego, CA) on an Illumina NextSeq 500 or a MiSeq sequencer (Illumina, San Diego, CA), generating 150-bp paired-end reads. Samples were aligned with the reference human genome GRCh37/hg19 and data analysis was performed as previously described [24] or using the BasespaceTruSeq ${ }^{\circledR}$ Amplicon App version 1.1 or own developed algorithms. Variant interpretation followed the American College of Medical Genetics and Genomics (ACMG) guidelines [25]. Mutations were confirmed by conventional Sanger sequencing.

Genetic variants are reported following official Human Genome Variation Sequence (HGVS) nomenclature and refer to NM_000096.3 for the Homo sapiens CP transcript variant, and NP_000087.1 for Homo sapiens ceruloplasmin precursor protein. Reported mutations in this study have been submitted 
to the Leiden Open Variation Database (http://www.lovd.nl) or to ClinVar (http://www.ncbi.nlm.nih. gov/clinvar) [26,27].

\subsection{Bioinformatics and Computational Analysis}

In silico predictions of missense variants, pathogenicity was performed using SIFT and Polyphen-2 bioinformatics tools [28,29].

Ceruloplasmin sequences from different species were retrieved from the PFAM database [30] and aligned using the MUSCLE program [31] for multiple alignments. Conservation analysis and alignment visualization were performed by Jalview software (version 2, www.jalview.org) [32].

The structural analysis of the missense variants was made based on the available human $\mathrm{CP}$ crystallographic structure [33]. Human Ceruloplasmin crystallographic structure was downloaded from the PDB server (PDB code: 4ENZ, crystallographic resolution: $2.6 \AA$; (http://www.rcsb.org/pdb/ home/home.do). The Consurf server was used to map conservation features on the structure [34]. The prediction of the putative effects of the variants in the structure/function of the protein was performed by visual inspection using the Chimera program [35]. The wild-type residues and the modeled mutants were included in the IronGenes database, publicly accessible from: http: $/ /$ molsim.sci.univr.it/marchetto/php/gene_detail.php?geneId=CP\#tabellaInit: from the first page, the results can be accessed graphically by selecting the 'Variants Structures and Models' button, and then directly exploring the different variants.

To analyze the plausible splicing effects of the CP c.1864+5G $>\mathrm{A}$ intronic variant, we used the bioinformatic NNSplice algorithm based on neural networks (http://www.fruitfly.org/seq_tools/splice. html [36]) and the Human Splicing Finder software [37] based on position-dependent logic.

\section{Results}

\subsection{Clinical and Biochemical Profile}

In this international collaborative study, we describe 11 new ACP families with 13 affected individuals (six men and seven women). Table 1 summarizes the biochemical, clinical, and genetic data of the patients. Pedigrees are shown in Figure 2. Family origins were spread worldwide, but none of the patients had Japanese ancestry (Table 1). Age at diagnosis ranged from 16 to 75 years (median 40 years), with an average diagnostic delay (i.e., time from clinical onset to the molecular diagnosis) of 10 years or more. 
Table 1. Biochemical, clinical, and genetic data of the 13 affected members in the 11 studied families with ACP.

\begin{tabular}{|c|c|c|c|c|c|c|c|c|c|c|c|c|c|c|}
\hline & Family 1 & Family 2 & Family 3 & Family 4 & Family 4 & Family 5 & Family 6 & Family 7 & Family 8 & Family 8 & Family 9 & Family 10 & Family 11 & Normal \\
\hline Characteristic & II.3 & II.3 & II.3 & II.2 (proband) & II.3 & II.1 & II. 2 & II.3 & II.3 (proband) & II. 2 & II. 2 & II. 1 & II.1 & Values \\
\hline Country of Origin & Lithuania & Spain & India & India & India & $\begin{array}{l}\text { Poland } \\
\end{array}$ & Italy & Italy & Brazil & Brazil & Brazil & India & Pakistan & \\
\hline Sex & F & $\mathrm{F}$ & $\mathrm{M}$ & $\mathrm{M}$ & $\mathrm{M}$ & F & $\mathrm{M}$ & $\mathrm{M}$ & F & F & F & $\mathrm{F}$ & $\mathrm{M}$ & \\
\hline Age at Dx (years) & 75 & 33 & 40 & 66 & 61 & 40 & 46 & 62 & 37 & 29 & 46 & 25 & 16 & \\
\hline $\mathrm{Hb}(\mathrm{g} / \mathrm{dl})$ & 11.1 & 10.9 & 12.1 & 11.6 & 10.7 & 11.1 & 12.5 & 12.2 & 11.7 & 12.3 & 9.4 & 9.2 & 13.4 & $12-16$ \\
\hline MCV (fl) & 82,0 & 85.1 & 77 & 66.8 & 81 & 88.0 & 84 & 70.6 & 75.2 & 71.4 & 64.5 & 71.5 & 69 & $79-99$ \\
\hline Retyculocytes $(\%)$ & 0.5 & 0.8 & $n / a$ & 0.9 & 1.2 & $n / a$ & 0.58 & 1 & 1.77 & 1.1 & 1.77 & 0.51 & n.a. & $1.1-2.7$ \\
\hline $\mathrm{RDW}(\%)$ & 16.8 & 15.2 & 14.3 & 17.3 & 15.5 & 15.5 & 15.4 & 16.1 & 16 & 17.2 & 18 & 17.1 & 18 & $11.3-14.5$ \\
\hline Serum iron ( $\mu \mathrm{g} / \mathrm{dl})$ & 82.8 & 15 & 28 & 19 & 39.1 & 81.0 & 33 & 215 & 23 & 23 & 22 & 9.5 & 33.5 & $37-170$ \\
\hline Ferritin $(\mathrm{n} g / \mathrm{ml})$ & 12159 & 355.4 & 1077 & 1112 & 3845 & 1143 & 2100 & 3650 & 791 & 732 & 1060 & 757 & 1065 & $10-290$ \\
\hline $\begin{array}{c}\text { Transferrin } \\
\text { Saturation }(\%)\end{array}$ & 12.4 & 4.3 & 10 & 5 & 12 & 39 & 9 & 88 & 9.24 & 9.83 & 8.2 & 4 & 8.8 & $20-55$ \\
\hline $\mathrm{CP}(\mathrm{mg} / \mathrm{dl})$ & $<0.02$ & $<2.0$ & $<0.03$ & $<0.03$ & $<0.03$ & 0.12 & undetectable & 0.12 & 11 & 9 & $<2$ & n.a. & $<0.02$ & $17-65$ \\
\hline ALT (U/I) & 55 & 12 & 32 & 24 & 36 & 19 & 37 & 113 & 26 & 179 & 37 & 24 & 87 & $14-36$ \\
\hline AST (U/I) & 43 & 17 & n.a & n.a & 24 & 16 & 20 & 80 & 12 & 82 & 29 & $\mathrm{n} / \mathrm{a}$ & $n / a$ & $8-40$ \\
\hline $\begin{array}{l}\text { Clue to ACP } \\
\text { diagnosis }\end{array}$ & $\begin{array}{c}\text { Low level of Cp. } \\
\text { No Cu urine } \\
\text { excretion } \\
\text { Hepatocellular } \\
\text { siderosisi Iron } \\
\text { deposition in basal } \\
\text { gannglia. } \\
\end{array}$ & $\begin{array}{c}\text { Low level of Cp. } \\
\text { Low serum Cu and } \\
\text { low urine Cu. } \\
\text { Hepatic iron } \\
\text { overlodad Iron } \\
\text { deposit in basal } \\
\text { ganglias. } \\
\end{array}$ & $\begin{array}{l}\text { Iron deposition at } \\
\text { brain MRII } \\
\text { Uneplained } \\
\text { hyperfertritinemia, } \\
\text { low CP }\end{array}$ & $\begin{array}{l}\text { Iron deposition at } \\
\text { brain MRII } \\
\text { Uneplained } \\
\text { hyperferritinemia, } \\
\text { low CP }\end{array}$ & $\begin{array}{l}\text { Iron deposition at } \\
\text { brain MRII } \\
\text { Unexplaind } \\
\text { hyperfertritinemia, } \\
\text { low CP }\end{array}$ & $\begin{array}{l}\text { Symptoms } \\
\text { (including tremor) } \\
\text { and very low CP }\end{array}$ & $\begin{array}{c}\text { unexplained } \\
\text { hyperferritinemia }\end{array}$ & $\begin{array}{c}\text { overexpressed HFE } \\
\text { Hemochromatosis, } \\
\text { supposed } \\
\text { additional } \\
\text { non-HEF } \\
\text { mutation(s) tested } \\
\text { with NGS } \\
\end{array}$ & $\begin{array}{c}\text { Iron deposition at } \\
\text { brain MRI }\end{array}$ & $\begin{array}{c}\text { Familial } \\
\text { investigation }\end{array}$ & $\begin{array}{l}\text { Low CP in } \\
\text { investigation of } \\
\text { iron-refractory } \\
\text { anaemia }\end{array}$ & $\begin{array}{l}\text { Hair loss, mild } \\
\text { executive } \\
\text { dysfunction on } \\
\text { formal } \\
\text { neurocognitive } \\
\text { assessment }\end{array}$ & low CP level & \\
\hline $\begin{array}{c}\text { Clinical } \\
\text { presentation } \\
\text { (symptoms and } \\
\text { signs) }\end{array}$ & $\begin{array}{l}\text { Moderate dementiaa } \\
\text { with prevalent } \\
\text { frontal features, } \\
\text { cerebellar ataxia, } \\
\text { oromandibular } \\
\text { dystonia, torsion of } \\
\text { the trunk, severe } \\
\text { chorea-athetosis } \\
\text { with choreiform } \\
\text { movements. Mild } \\
\text { type-2 DM. Retinal } \\
\text { degeneration. Mild } \\
\text { anaemi }\end{array}$ & 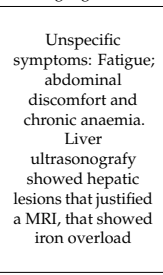 & 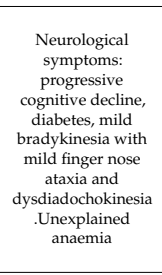 & 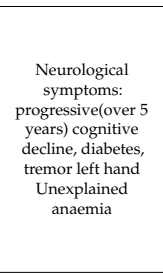 & $\begin{array}{l}\text { Concentration and } \\
\text { intellectual ability } \\
\text { decline. } \\
\text { Unexplained } \\
\text { anaemia, DM. }\end{array}$ & $\begin{array}{l}\text { Head and postural } \\
\text { tremorof of upper } \\
\text { and lower limbss, } \\
\text { slight dysmetria, } \\
\text { ataxia, proximal } \\
\text { weakness of olower } \\
\text { extremitites, } \\
\text { horizontal } \\
\text { nystamus, and } \\
\text { tunnel vision. }\end{array}$ & $\begin{array}{l}\text { Liver iron overload } \\
\text { and mild anaemia }\end{array}$ & $\begin{array}{l}\text { Iron overload. Mild } \\
\text { anaemia was } \\
\text { consistent also with } \\
\text { b-thalassemia trait }\end{array}$ & $\begin{array}{c}\text { Choreiform } \\
\text { movement disorder, } \\
\text { mild anaemia, } \\
\text { DM-2, } \\
\text { assmptomatic } \\
\text { retina pigmentation }\end{array}$ & $\begin{array}{c}\text { Asymptomatic. } \\
\text { Familial } \\
\text { investigation }\end{array}$ & $\begin{array}{l}\text { Mild anaemia, } \\
\text { DM-2, asthenia, } \\
\text { mild movement } \\
\text { disorder }\end{array}$ & 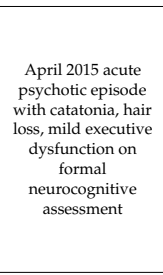 & $\begin{array}{l}\text { Concentration/memor } \\
\text { lapses }\end{array}$ & \\
\hline Anaemia & Yes & Yes & Yes & Yes & Yes & Yes & Yes & Yes & Yes & No & Yes & Yes & No & \\
\hline $\begin{array}{c}\begin{array}{c}\text { Neurological } \\
\text { symptoms }\end{array} \\
\text { symats }\end{array}$ & Yes & No & Yes & Yes & Yes & Yes & No & No & Yes & No & Yes & Yes & Yes & \\
\hline Liver Iron overload & Yes & Yes & n.a. & Yes & Yes & No & Yes & n.a. & n.a. & Yes & n.a & Yes & Yes & \\
\hline Diabetes & Yes & No & Yes & Yes & Yes & No & No & No & Yes & No & Yes & No & No & \\
\hline Retinopathy & Yes & № & No & No & No & Not evaluated & $\begin{array}{l}\text { Yes but not typical } \\
\text { for } \\
\text { aceruloplasminemia }\end{array}$ & No & Yes & Not evaluated & Not evaluated & No & Yes & \\
\hline $\begin{array}{l}\text { Brain MRI (sites of } \\
\text { iron accumulation, } \\
\text { in brief) }\end{array}$ & $\begin{array}{l}\text { Iron overload in } \\
\text { putamen }\end{array}$ & $\begin{array}{l}\text { Iron overload in } \\
\text { lenticular, dentate } \\
\text { and thalamus }\end{array}$ & $\begin{array}{l}\text { SWI increased } \\
\text { susceptibility } \\
\text { involving the } \\
\text { cerebelum, basal } \\
\text { ganglia, thalami, } \\
\text { red and dentate } \\
\text { nuclei. }\end{array}$ & $\begin{array}{l}\text { MRI SWI with } \\
\text { marked } \\
\text { susceptibility } \\
\text { predominantly } \\
\text { involving the } \\
\text { lateral putamen, } \\
\text { red nucleus, } \\
\text { striaum, thalamic, } \\
\text { pulvinat, cerebellar } \\
\text { dentate nucleus }\end{array}$ & $\begin{array}{l}\text { Iron overload in } \\
\text { lentiform caudate, } \\
\text { dorsal lateral } \\
\text { thalami and } \\
\text { dentate nuclei }\end{array}$ & $\begin{array}{l}\text { FLAIR and T2 } \\
\text { hypointenseties in } \\
\text { the putamen and } \\
\text { substantia nigra }\end{array}$ & no iron overload & no iron overload & $\begin{array}{l}\text { Iron overload in } \\
\text { thalami, basal } \\
\text { ganglia, and } \\
\text { cerebellum }\end{array}$ & $\begin{array}{l}\text { Iron overload in } \\
\text { thalami, abala } \\
\text { ganglia, red nuclei, } \\
\text { dentate, cerebelllum } \\
\text { and brain cortex }\end{array}$ & $\begin{array}{l}\text { Iron overload in } \\
\text { thalami, basal } \\
\text { ganglia, dentate, } \\
\text { and cerebellum }\end{array}$ & $\begin{array}{c}\text { Irono overload in } \\
\text { choroid plexus, } \\
\text { bilatera dentate } \\
\text { nuclei thalamic and } \\
\text { basal ganglia }\end{array}$ & n.a. & \\
\hline $\begin{array}{c}\text { Liver MRI or } \\
\text { (biopsy) }\end{array}$ & $\begin{array}{l}\text { hepatocellular } \\
\text { siderosis grade III }\end{array}$ & $\begin{array}{c}\mathrm{HIII}=9.58, \text { severe } \\
\text { iron overload }\end{array}$ & n.a. & $\begin{array}{l}\text { Feriscan LIC: } 9.4 \\
\text { mglg dw }\end{array}$ & $\begin{array}{l}\text { LLC by Ferriscan: } \\
6.3 \mathrm{mg} / \mathrm{d} \mathrm{dw}\end{array}$ & $\begin{array}{c}\text { Biopsy: small } \\
\text { depositions of } \\
\text { yellow-brown } \\
\text { pigment (stain for } \\
\text { ferrum- negative)- } \\
\text { probably lipofuscin }\end{array}$ & $\begin{array}{l}\text { LIC } 340 \mu \mathrm{M} / \mathrm{g}=\mathrm{HII} \\
7.9 \text { (severe iron } \\
\text { overload) }\end{array}$ & n.a. & not performed & $\begin{array}{l}\text { Iron overload in } \\
\text { liver and pancreas } \\
\text { (qualitative) }\end{array}$ & not performed & $\begin{array}{l}\text { LLC by Ferriscan } \\
9.0 \mathrm{mg} / \mathrm{d} / \mathrm{dw}\end{array}$ & $\begin{array}{l}\text { LIC by Ferrican: } \\
5.3 \mathrm{mg} / \mathrm{g} \mathrm{dw}\end{array}$ & HIII $<1.9$ \\
\hline Diagnostic delay & 20 years & 1.5 years & 5 years & 2 years & 4 years & 13 years & 3 years & 30 years & 1 year & Not applicable & 32 years & 3 years & Not known & \\
\hline
\end{tabular}


Table 1. Cont.

\begin{tabular}{|c|c|c|c|c|c|c|c|c|c|c|c|c|c|c|}
\hline Characteristic & $\begin{array}{ll}\text { Family } 1 \\
\end{array}$ & $\begin{array}{c}\text { Family } 2 \\
\end{array}$ & Family 3 & Family 4 & $\begin{array}{l}\text { Family } 4 \\
\end{array}$ & Family 5 & Family 6 & $\begin{array}{l}\text { Family } 7 \\
\end{array}$ & Family 8 & $\begin{array}{ll}\text { Family } 8 \\
\end{array}$ & $\begin{array}{l}\text { Family } 9 \\
\end{array}$ & Family 10 & Family 11 & $\begin{array}{c}\text { Normal } \\
\text { Vomat }\end{array}$ \\
\hline Cnaractersictc & 11.3 & II.3. & II. 3 & III.2 (proband) & 11.3 & II.1. & II. 2 & 11.3 & 11.3 (proband) & II.2 & 11.2 & II.1. & II.1. & \\
\hline $\begin{array}{l}\text { Iron chelation or } \\
\text { other therapy }\end{array}$ & No Iron chelation therapy. & 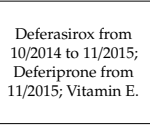 & $\begin{array}{l}\text { Started on FFP } \\
\text { OctaplasLG every } 2 \\
\text { weeks on diagnosis. } \\
\text { Later added } \\
\text { Deferiprone } \\
\end{array}$ & $\begin{array}{l}\text { Deferiprone started } \\
\text { on diagnosisis }(25 \\
m g / k g / d)\end{array}$ & $\begin{array}{l}\text { Deferiprone started } \\
2011\end{array}$ & Zinc & $\begin{array}{c}\text { Deferasisiox and } \\
\text { desferoxamine both } \\
\text { suspended for } \\
\text { renal insufficency, } \\
\text { actually on } \\
\text { deferiprone } \\
\end{array}$ & $\begin{array}{l}\text { Desferoxamine and } \\
\text { "micro"-phlebotomies }\end{array}$ & Deferiprone & $\begin{array}{l}\text { Desferoxamine, } \\
\text { combination with } \\
\text { deferiprone }\end{array}$ & Deferiprone & Deferiprone & & \\
\hline $\begin{array}{l}\text { Genetics CP gene } \\
\text { NM_000096.3; } \\
\text { NP__000087.1 }\end{array}$ & 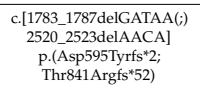 & 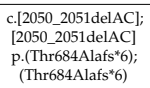 & $\begin{array}{l}\text { c. }[1864+5 \mathrm{G}>\mathrm{A}] ; \\
{[1864+5 \mathrm{G}>\mathrm{A}]}\end{array}$ & $\begin{array}{l}\text { c. }[1864+5 G>A] \\
{[1864+5 G>A]}\end{array}$ & $\begin{array}{c}\text { c. }[1864+ \\
5 \mathrm{G}>\mathrm{A}][11864+5 \mathrm{G}>\mathrm{A}]\end{array}$ & 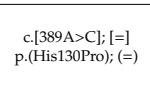 & 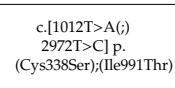 & 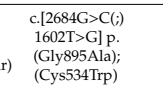 & $\begin{array}{l}c .12879-1 G>T] ; \\
{[2879-1 G>T]}\end{array}$ & $\begin{array}{l}c .[2879-1 \mathrm{G}>\mathrm{T}] \\
{[2879-1 \mathrm{G}>\mathrm{T}]}\end{array}$ & 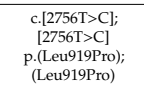 & 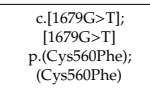 & $\begin{array}{l}\text { c. [1713+1delG]; } \\
\text { [1713+1delG] }\end{array}$ & \\
\hline
\end{tabular}

Reference values are indicated in the last column. Sex: M: male, F: female; ACP: Aceruloplasminemia; CP: ceruloplasmin; MRI: magnetic resonance imaging; SWI Susceptibility weighted imaging; HII hypoxic-ischemic injury; LIC: liver iron content; Hb: Hemoglobin; MCV: mean corpuscular volume; RDW: red cell distribution width; DM-2: Diabetes mellitus type 2; NGS: next-generation sequencing; n.a.: not available. 

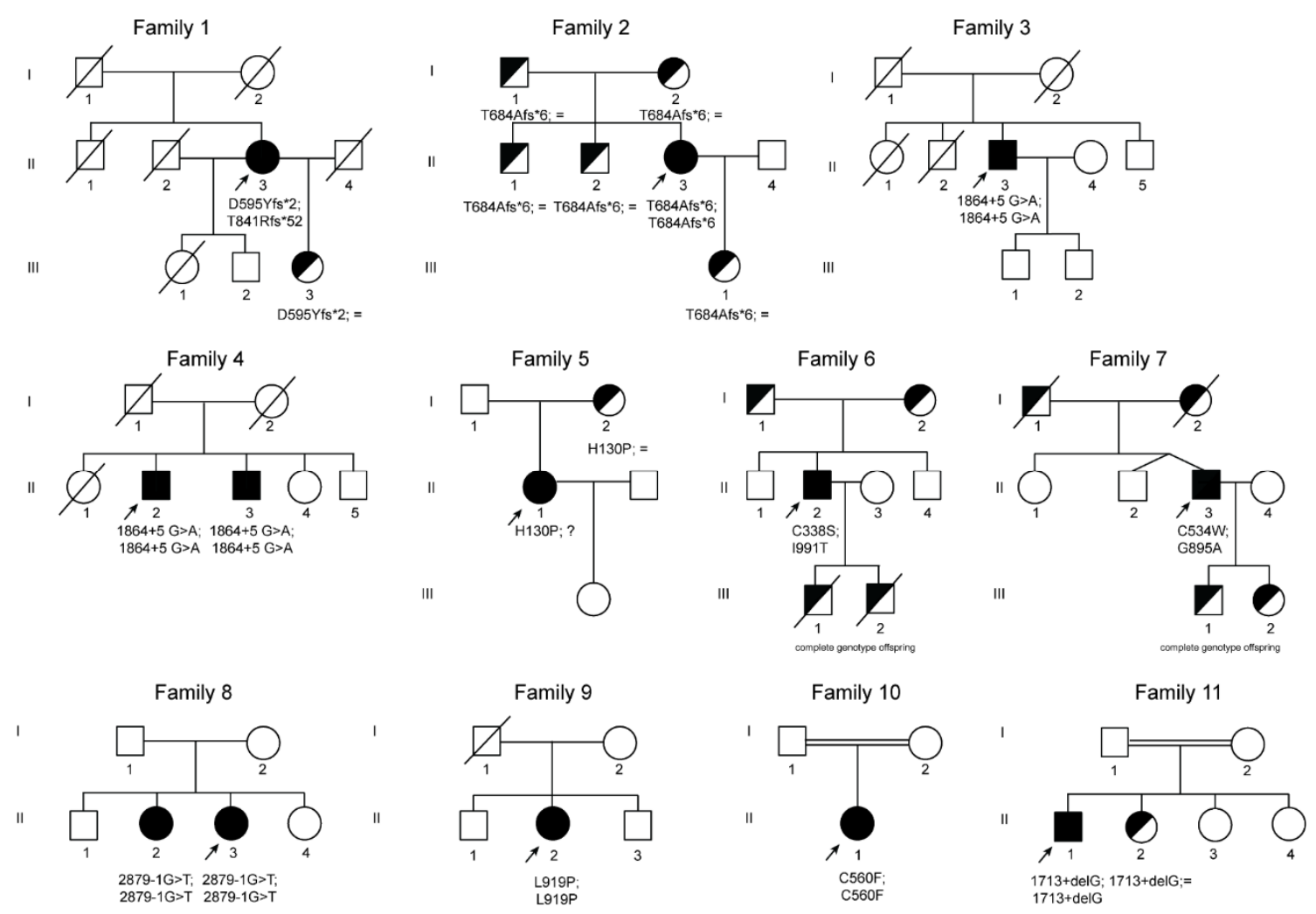

Figure 2. Pedigree trees from 11 studied families affected by ACP. Squares indicate males and circles females. Index cases are indicated with an arrow. Filled symbols indicate affected members, half-filled black symbols denote unaffected carriers, and barred symbols indicate deceased subjects. "?", indicates individuals that were not possible to test because of refusal of testing or non-locatable person. Mutations are named according to the Human Genome Variation Sequence (HGVS) nomenclatures.

In our cohort, 11/13 (84.6\%) patients had anemia (mild in 9/11), and 8/13 had microcytosis (61,5\%). In all patients, serum ferritin values were high (geometrical mean $1409 \mathrm{ng} / \mathrm{mL}, \mathrm{CI} 95 \%$ 809.5-2452.5), and ceruloplasmin levels were very low or even undetectable. TSAT was decreased in 11/13 patients $(84.6 \%)$, and serum iron was decreased in $10 / 13$ patients $(76.9 \%)$. Neurological symptoms were present in $75 \%$ of patients (data available from $12 / 13$ patients) and ranged from severe complex neurological and psychiatric syndromes to mild cognitive dysfunctions. Brain iron overload at MRI was proven in ten $(83.3 \%)$ of such patients. Eight out of nine patients (88.9\%) had liver iron overload assessed by either liver biopsy (Figure 1) or MRI. Diabetes mellitus and retinopathy were present in $46.2 \%$ and $40 \%$ of cases, respectively. All patients are alive since our last contact with them.

\subsection{Genetic Spectrum}

Using a combination of Sanger sequencing and NGS, we identified 13 unique mutations, 10 of which are novel (see details in supplementary data).

Three novel frameshift mutations were detected in family 1 and family 2 (Figure 2, Table 1). Patient (II.3) from family 1 is compound heterozygous for two frameshift variants, the c.1783_1787delGATAA and c.2520_2523delAACA located in exon 10 and 14 of the $C P$ gene, respectively. Both mutations lead to the creation of aberrant C-terminal CP protein ends and premature stop codons at position 596 and 892, respectively p.(Asp595Tyrfs*2 and Thr841Argfs*52). Proband (II.3) from family 2 has a novel homozygous deletion at position 2050 to 2051 of the $C P$ gene leading to a premature stop codon at position 689 of ceruloplasmin protein p.(Thr684Alafs*6).

In families 3 and 4 , sequencing analysis revealed in all probands and in one relative (brother of patient II. 1 from family 4) a novel homozygous intronic mutation at position +5 downstream exon 10 
of the $C P$ gene (Figure 2 and Table 1). All individuals presenting this mutation are from India. The NNSplice algorithm program and the Human Splicing Finder splicing program predicted that the substitution of a $G$ by an $A$ at position +5 will abolish the wild-type donor splicing site of intron 10 , interfering with $C P$ splicing.

In family 5, a new missense mutation was detected in heterozygous state p.(His130Pro). His130 is engaged in a hydrogen bond with Tyr155, and its substitution with a proline causes the loss of this interaction (http://molsim.sci.univr.it/marchetto/php/gene_detail.php?geneId=CP\#tabellaInit). Although this histidine is not well conserved in our multiple sequence alignment (Figure S1), in this position, there is always a polar or charged residue present, indicating that a substitution with a hydrophobic residue could cause protein misfolding. In this patient, we cannot exclude the existence of an additional mutation in non-studied regions (i.e., deep intronic or promoter regions). Interestingly, additional ACP cases with only one mutation and a second wild-type allele have also been previously described in the literature (see Table S1).

In family 6, the proband (II.2) is compound heterozygous for two CP missense mutations, p.(Cys338Ser) and p.(Ile991Thr). These two variants have been recently categorized as pathogenic mutations in ACP cases [38]. Indeed, Cys338 forms the binding cavity for one of the $\mathrm{Cu}^{2+}$ ions (Figure 3 and http://molsim.sci.univr.it/marchetto/php/gene_detail.php?geneId=CP\#tabellaInit), and thus, the lack of a cysteine residue in that position could dramatically affect protein function.

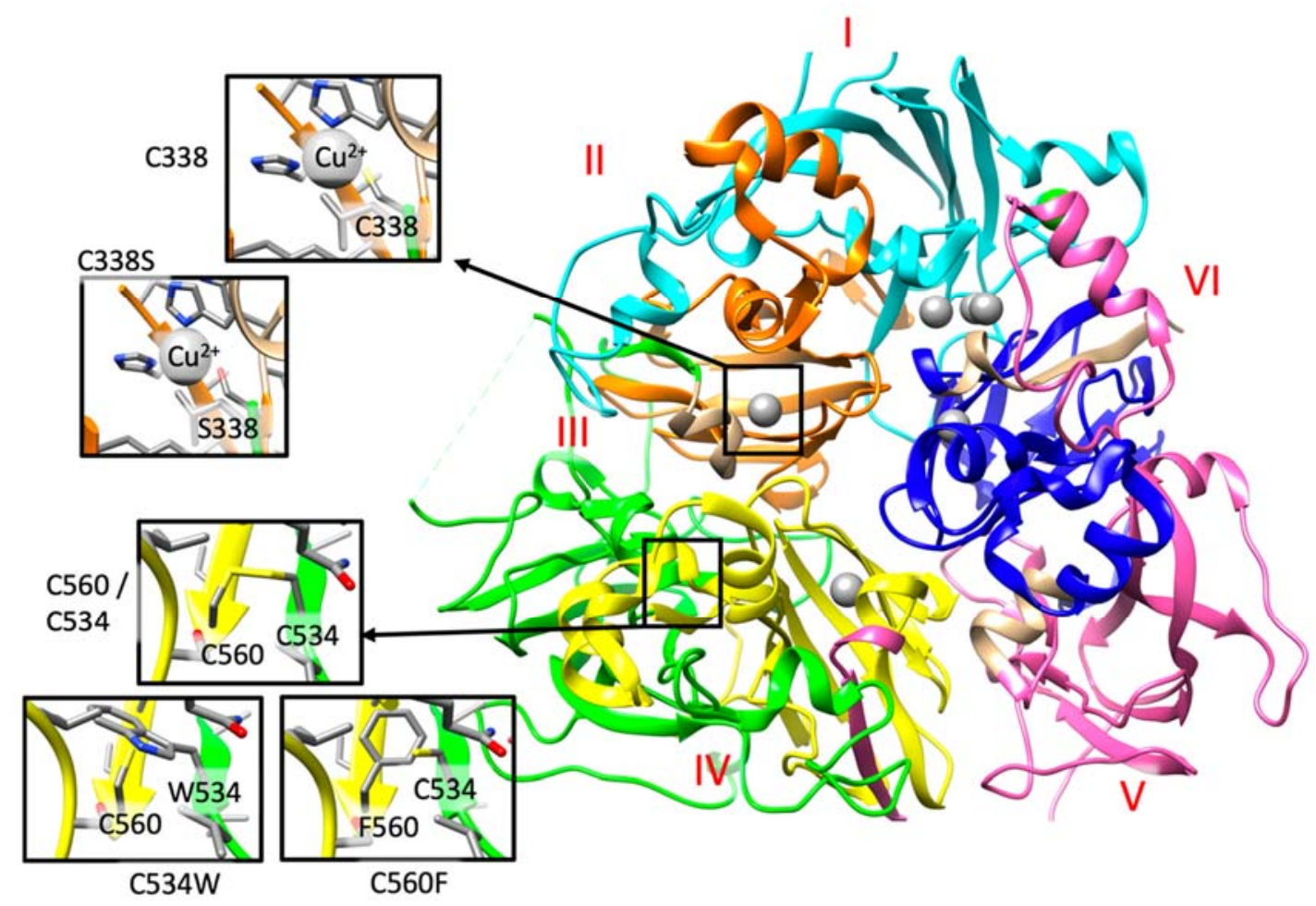

Figure 3. A. Detail of the structural analysis of selected missense mutations mapped on the structure of human ceruloplasmin (PDB code: 4ENZ). The reference and the mutated residue are indicated within the insights. The six Plastocyanin-like domains, described in the Uniprot Database (https: //www.uniprot.org/uniprot/P00450) I to VI are colored in cyan, orange, green, yellow, pink, and blue, respectively. Copper domains are colored in grey. These three variants, as well as those detailed in the main text, can be retrieved from the IronGenes database (http://molsim.sci.univr.it/marchetto/php/ gene_detail.php?geneId=CP\#tabellaInit) developed at the University of Verona.

In family 7, the patient is compound heterozygous for the missense mutation p.(Gly895Ala) (rs139633388, previously reported as p.(Gly876Ala) due to alternate nomenclature) and a novel missense variant, p.(Cys534Trp). The Gly895 is an evolutionary highly conserved (Figure S1) amino acid, and 
in silico modeling analysis indicated that its substitution by alanine could cause local perturbations due to size differences. Cys534 residue is involved in a disulfide bridge with Cys560 (Figure 2 and http://molsim.sci.univr.it/marchetto/php/gene_detail.php?geneId=CP\#tabellaInit), and both cysteines are highly conserved (Figure S1); therefore, the p.(Cys534Trp) variant is predicted to have a high impact on protein stability.

In family 8 , the proband and her elder sister carries a new splicing mutation (c.2879-1 G>T) in homozygosity in the donor site of intron 16 (variant rs386134141 and ClinVar ID 431112 reported by us). This mutation is predicted to lead to the loss of the splicing donor site located upstream of exon 17. A pathogenic mutation in the same position but with the change G>A (c. 2879-1 G>A, reported as nt2878-1G/A) was previously described as causative of ACP (see supplementary Table S1 and supplementary Reference 4).

In family 9, the proband is homozygous for a new missense mutation p.(Leu919Pro) in exon 16 (variant rs1135401784 and ClinVar ID 431113 reported by us). According to our computational model, Leu919 is involved in a network of hydrophobic interactions with Leu921 and Leu808 (http://molsim.sci.univr.it/marchetto/php/gene_detail.php?geneId=CP\#tabellaInit) and is conserved in $86 \%$ of the sequences (Figure S1). The substitution by a proline would probably lead to perturbations in this network, altering the stability of the protein.

In family 10, the proband (II.1) is homozygous for a new missense mutation p.(Cys560Phe) in exon 9. Interestingly, Cys560 is 100\% conserved (Figure S1) and is engaged in a disulfide bridge with Cys534; therefore, its substitution by Phe would have a high impact on the protein stability and/or structure (Figure 3 and http://molsim.sci.univr.it/marchetto/php/gene_detail.php?geneId=CP\#tabellaInit). The effect, breaking of a disulfide bridge, is the same as the one encountered for the mutant Cys534 in family 7 , pinpointing to a critical functional region of the protein.

In family 11, the proband (II.1) is homozygous for a new splicing mutation in intron 9, c.1713+1delG, which alters the donor splice site of exon 9, which is predicted to result in the skipping of exon 9. His cousin has the same phenotype and his sister is a carrier of this splicing mutation.

In Table S1, we provide an updated overview of all ACP cases reported in the literature and, if available, the corresponding CP mutations. Including our cohort, 107 patients with ACP from 83 families with a worldwide distribution were identified. Most reported cases are of Japanese origin, followed by Italian cases. In total, there are 71 different reported mutations causing ACP, distributed along the $\mathrm{CP}$ gene. All types of mutations have been reported (13 splicing mutations, 28 missense, 8 nonsense, 19 frameshift, and 2 big deletions), including 10 novel mutations that we reported in this study (3 splicing, 4 missense, and 3 frameshift mutations) (Figure S2). Most CP mutations are restricted to a particular family.

\section{Discussion}

To the best of our knowledge, this study represents the largest series of non-Japanese ACP patients reported so far. The median age at diagnosis was 40 years, and the median diagnostic delay was about 10 years. Clinical and biochemical features of patients highlight substantial phenotypic heterogeneity, as also reported by a recent Italian case series [38], which contributes to the difficulties in the diagnosis of such a rare disease. The complete clinical triad of retinal degeneration, dementia, and diabetes, cited in a previous Japanese series [39], was present only in one 79-year-old patient (proband II.3 from family 1). Our data support that unexplained anemia, often but not necessarily microcytic, in association with low TSAT and paradoxically high ferritin are the best clues for an early diagnosis, which is crucial to prevent irreversible neurological manifestations. This type of anemia can be defined as "atypical microcytic anemia" to differentiate from other common causes of microcytosis, such as iron deficiency anemia or thalassemias [40-42]. With the exception of iron-refractory iron-deficiency anemia (IRIDA), atypical microcytic anemias are accompanied by progressive iron overload. In this regard, we propose a simplified algorithm for the diagnosis of ACP starting from microcytic anemia and hyperferritinemia in Figure 4. 


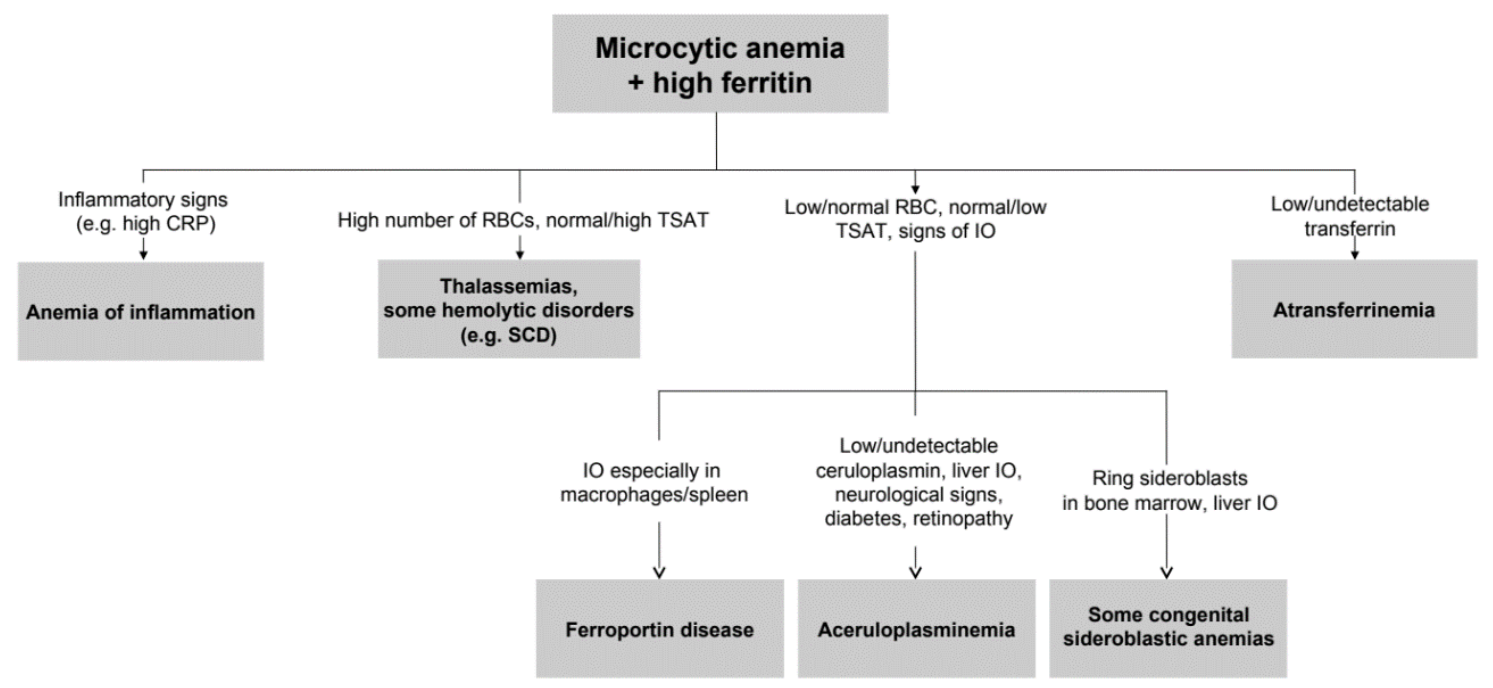

Figure 4. Simplified approach to microcytic anemia + high ferritin. From relatively simple laboratory tests also rare diseases can be suspected. Further investigations should include genetic testing, MRI for estimation of liver iron content, and eventually liver or bone marrow histopathology. IO: iron overload. SCD: sickle cell disease.

In our series, neurological involvement and diabetes were present in $75 \%$ and $46.2 \%$ of patients, respectively, substantially in line with a previous case series $[38,43,44]$. Retinopathy was described in $40 \%$ of our patients, while it is reported in $76 \%$ of Japanese ACP patients [44]. Additional sporadic clinical findings in our series included hypothyroidism, amenorrhea, abdominal pain, and chronic diarrhea, but it was not possible to prove a causative role of $\mathrm{ACP}$, which remains uncertain.

A genetic study of our patients also confirmed genetic heterogeneity. We detected 13 unique mutations, 10 of which are novel and distributed throughout the entire gene. Indeed, the aminoacidic changes affected all the six structural domains, with presumable different functional effects. The in-silico modeling provided in our work (and available at the link http://molsim.sci.univr.it/marchetto/ php/gene_detail.php?geneId=CP\#tabellaInit) is an attempt to further clarify the functional role of these mutations.

A description of new mutations, their functional study, and genotype-phenotype correlations are important for several reasons in $\mathrm{ACP}$, including elucidate pathophysiology, understand why some patients develop brain iron accumulation and others do not, help in understanding brain iron metabolism also in other more common neurodegenerative disorders, and hopefully help in developing new and more efficient treatments.

\section{Conclusions}

Our study points out that ACP is distributed worldwide and is characterized by heterogeneous genotypes and phenotypes, requiring a high level of suspicion for diagnosis. Atypical anemia, often with microcytosis, low TSAT, and high ferritin, is the best clue for early pre-symptomatic diagnosis, which is crucial to avoid treatment delay and the onset of irreversible neurological damage. Collaborative joint efforts are needed among specialized laboratories and centers, as in our work, for a better definition of the molecular and clinical features of ACP, hopefully leading to new pathophysiological and therapeutical insights for this potentially incapacitating disease.

Supplementary Materials: Supplementary materials can be found at http://www.mdpi.com/1422-0067/21/7/2374/ s1.

Author Contributions: M.S., M.V.C., G.M., A.B. and D.G., designed, analyzed the data, and wrote the manuscript. M.S., C.E.-J., genetic studies in P1, P2, P3, P4, and P5. B.B., P1 clinical and hematological characterization. A.U. and G.K., P1 neurological clinical data. E.J.-B., J.S.-D., N.B.-D., P2 clinical and hematological characterization, V.C., H.H., N.W.W., P3, and P4 clinical and hematological characterization; K.K. and B.K., P5 clinical and hematological 
characterization. D.G., F.B. and G.M., P6 and P7 clinical and hematological characterization. C.P., genetic studies and data analysis in P6 and P7. E.S., A.M. and A.G., computational in silico modeling studies for all missense mutations. M.D.B., D.M.d.A., P9 and P10 genetic studies, K.Y.F., P9 and P10 clinical and hematological characterization, data analysis. P.B., P4, P10, and P11 genetic studies. J.B.P. and P.E., P4 and P10 clinical and hematological characterization. S.P., P11 clinical and hematological characterization. All authors have read and agreed to the published version of the manuscript.

Funding: This work was partially supported by grant SAF2015-70412-R from the Spanish Secretary of Research, Development, and Innovation (MINECO) Spain to M.S., grants for research on iron metabolism by Fondazione Cariverona (2014.0851), and the Veneto Region (PRIHTA no.2014-00000451) to D.G., grants 2014/00984-3 and 2016/08072-9 from Fundação de Apoio à Pesquisa do Estado de São Paulo (FAPESP) to K.Y.F. and M.D.B., respectively.

Acknowledgments: The authors would like to thank all patients and their family members for their participation in this study. We thank Alejandro Negro and Jessica Aranda from Institute Josep Carreras and Josep Fita from BloodGenetics for their technical support with Sanger and NGS sequencing. We thank Paola Capelli, at the Pathology Institute of the University Hospital of Verona for providing the histopathologic figure. We are grateful to The Spastic Paraplegia Foundation, The Brain Research Trust (BRT), The UK HSP Society, The Medical Research Council (MRC UK MR/J004758/1,G0802760,G1001253), The Wellcome Trust (equipment and the Synaptopathies strategic award (104033)), Rosetrees Trust, Ataxia UK and British Neurological Surveillance Unit (BNSU). We are also supported by the National Institute for Health Research (NIHR), University College London Hospitals (UCLH) and Biomedical Research Centre (BRC).

Conflicts of Interest: The authors declare no competing financial interests. The funders had no role in the design of the study; in the collection, analyses, or interpretation of data; in the writing of the manuscript, or in the decision to publish the results.

\section{References}

1. Miyajima, H. Aceruloplasminemia. Neuropathology 2015, 35, 83-90. [CrossRef] [PubMed]

2. Marchi, G.; Busti, F.; Lira Zidanes, A.; Castagna, A.; Girelli, D. Aceruloplasminemia: A Severe Neurodegenerative Disorder Deserving an Early Diagnosis. Front. Neurosci. 2019, 13, 325. [CrossRef] [PubMed]

3. Miyajima, H.; Nishimura, Y.; Mimguchi, K.; Sakamoto, M.; Shimizu, T.; Honda, N. Familial apoceruloplasmin deficiency associated with blepharospasm and retinal degeneration. Neurology 1987, 37, 761-767. [CrossRef] [PubMed]

4. Miyajima, H.; Takahashi, Y.; Kamata, T.; Shimizu, H.; Sakai, N.; Gitlin, J.D. Use of desferrioxamine in the treatment of aceruloplasminemia. Ann. Neurol. 1997, 41, 404-407. [CrossRef] [PubMed]

5. Skidmore, F.M.; Drago, V.; Foster, P.; Schmalfuss, I.M.; Heilman, K.M.; Streiff, R.R. Aceruloplasminaemia with progressive atrophy without brain iron overload: Treatment with oral chelation. J. Neurol. Neurosurg. Psychiatry 2008, 79, 467-470. [CrossRef]

6. Piperno, A.; Alessio, M. Aceruloplasminemia: Waiting for an Efficient Therapy. Front. Neurosci. 2018, 12, 903. [CrossRef]

7. Yonekawa, M.; Okabe, T.; Asamoto, Y.; Ohta, M. A case of hereditary ceruloplasmin deficiency with iron deposition in the brain associated with chorea, dementia, diabetes mellitus and retinal pigmentation: Administration of fresh-frozen human plasma. Eur. Neurol. 1999, 42, 157-162. [CrossRef]

8. Kuhn, J.; Bewermeyer, H.; Miyajima, H.; Takahashi, Y.; Kuhn, K.F.; Hoogenraad, T.U. Treatment of symptomatic heterozygous aceruloplasminemia with oral zinc sulphate. Brain Dev. 2007, 29, 450-453. [CrossRef]

9. Zanardi, A.; Conti, A.; Cremonesi, M.; D’Adamo, P.; Gilberti, E.; Apostoli, P.; Cannistraci, C.C.; Piperno, A.; David, S.; Alessio, M. Ceruloplasmin replacement therapy ameliorates neurological symptoms in a preclinical model of aceruloplasminemia. EMBO Mol. Med. 2018, 10, 91-106. [CrossRef]

10. Wang, B.; Wang, X.P. Does Ceruloplasmin Defend Against Neurodegenerative Diseases? Curr. Neuropharmacol. 2019, 17, 539-549. [CrossRef]

11. Bento, I.; Peixoto, C.; Zaitsev, V.N.; Lindley, P.F. Ceruloplasmin revisited: Structural and functional roles of various metal cation-binding sites. Acta Crystallogr. D Biol. Crystallogr. 2007, 63 Pt 2, 240-248. [CrossRef]

12. Sato, M.; Gitlin, J.D. Mechanisms of copper incorporation during the biosynthesis of human ceruloplasmin. J. Biol. Chem. 1991, 266, 5128-5134. 
13. Patel, B.N.; Dunn, R.J.; David, S. Alternative RNA splicing generates a glycosylphosphatidylinositol-anchored form of ceruloplasmin in mammalian brain. J. Biol. Chem. 2000, 275, 4305-4310. [CrossRef] [PubMed]

14. Kono, S.; Yoshida, K.; Tomosugi, N.; Terada, T.; Hamaya, Y.; Kanaoka, S.; Miyajima, H. Biological effects of mutant ceruloplasmin on hepcidin-mediated internalization of ferroportin. Biochim. Biophys. Acta 2010, 1802, 968-975. [CrossRef] [PubMed]

15. Drakesmith, H.; Nemeth, E.; Ganz, T. Ironing out Ferroportin. Cell Metab. 2015, 22, 777-787. [CrossRef] [PubMed]

16. Muckenthaler, M.U.; Rivella, S.; Hentze, M.W.; Galy, B. A Red Carpet for Iron Metabolism. Cell 2017, 168, 344-361. [CrossRef] [PubMed]

17. Kenawi, M.; Rouger, E.; Island, M.L.; Leroyer, P.; Robin, F.; Remy, S.; Tesson, L.; Anegon, I.; Nay, K.; Derbre, F.; et al. Ceruloplasmin deficiency does not induce macrophagic iron overload: Lessons from a new rat model of hereditary aceruloplasminemia. FASEB J. 2019, 33, 13492-13502. [CrossRef]

18. Aschemeyer, S.; Qiao, B.; Stefanova, D.; Valore, E.V.; Sek, A.C.; Ruwe, T.A.; Vieth, K.B.; Jung, G.; Carla, C.; Rivella, S.; et al. Structure-function analysis of ferroportin defines the binding site and an alternative mechanism of action of hepcidin. Blood 2018, 131, 899-910. [CrossRef]

19. Kono, S. Aceruloplasminemia: An update. Int. Rev. Neurobiol. 2013, 110, 125-151.

20. Nemeth, E.; Tuttle, M.S.; Powelson, J.; Vaughn, M.B.; Donovan, A.; Ward, D.M.; Ganz, T.; Kaplan, J. Hepcidin regulates cellular iron efflux by binding to ferroportin and inducing its internalization. Science 2004, 306, 2090-2093. [CrossRef]

21. Ganz, T. Systemic iron homeostasis. Physiol. Rev. 2013, 93, 1721-1741. [CrossRef] [PubMed]

22. Hellman, N.E.; Gitlin, J.D. Ceruloplasmin metabolism and function. Annu. Rev. Nutr. 2002, 22, 439-458. [CrossRef] [PubMed]

23. Piubelli, C.; Castagna, A.; Marchi, G.; Rizzi, M.; Busti, F.; Badar, S.; Marchetti, M.; de Gobbi, M.; Roetto, A.; Xumerle, L.; et al. Identification of new BMP6 pro-peptide mutations in patients with iron overload. Am. J. Hematol. 2017, 92, 562-568. [CrossRef] [PubMed]

24. Badar, S.; Busti, F.; Ferrarini, A.; Xumerle, L.; Bozzini, P.; Capelli, P.; Giorgetti, A. Identification of novel mutations in hemochromatosis genes by targeted next generation sequencing in Italian patients with unexplained iron overload. Am. J. Hematol. 2016, 91, 420-425. [CrossRef]

25. Richards, S.; Aziz, N.; Bale, S.; Bick, D.; Das, S.; Gastier-Foster, J.; Geody, W.W.; Hegde, M.; Lyon, E.; Spector, E.; et al. Standards and guidelines for the interpretation of sequence variants: A joint consensus recommendation of the American College of Medical Genetics and Genomics and the Association for Molecular Pathology. Genet. Med. 2015, 17, 405-424. [CrossRef]

26. Sakamoto, H.; Inoue, K.; Higuchi, I.; Ono, Y.; Shimura, Y. Control of Drosophila Sex-lethal pre-mRNA splicing by its own female-specific product. Nucleic Acids Res. 1992, 20, 5533-5540. [CrossRef]

27. Joshi, R.; Shvartsman, M.; Morán, E.; Lois, S.; Aranda, J.; Barqué, A.; de la Cruz, X.; Bruguera, M.; Vagace, J.M.; Gervasini, G.; et al. Functional consequences of transferrin receptor-2 mutations causing hereditary hemochromatosis type 3. Mol. Genet. Genom. Med. 2015, 3, 221-232. [CrossRef]

28. Adzhubei, I.A.; Schmidt, S.; Peshkin, L.; Ramensky, V.E.; Gerasimova, A.; Bork, P.; Kondrashov, A.S.; Sunyaev, S.R. A method and server for predicting damaging missense mutations. Nat. Methods 2010, 7 , 248-249. [CrossRef]

29. Kumar, P.; Henikoff, S.; Ng, P.C. Predicting the effects of coding non-synonymous variants on protein function using the SIFT algorithm. Nat. Protoc. 2009, 4, 1073-1081. [CrossRef]

30. Finn, R.D.; Mistry, J.; Tate, J.; Coggill, P.; Heger, A.; Pollington, J.E.; Gavin, O.L.; Gunasekaran, P.; Ceric, G.; Forslund, K.; et al. The Pfam protein families database. Nucleic Acids Res. 2010, 38, D211-D222. [CrossRef]

31. Edgar, R.C. MUSCLE: Multiple sequence alignment with high accuracy and high throughput. Nucleic Acids Res. 2004, 32, 1792-1797. [CrossRef] [PubMed]

32. Waterhouse, A.M.; Procter, J.B.; Martin, D.M.; Clamp, M.; Barton, G.J. Jalview Version 2-a multiple sequence alignment editor and analysis workbench. Bioinformatics 2009, 25, 1189-1191. [CrossRef]

33. Samygina, V.R.; Sokolov, A.V.; Bourenkov, G.; Petoukhov, M.V.; Pulina, M.O.; Zakharova, E.T.; Vasilyev, V.B.; Bartunik, H.; Svergun, D.I. Ceruloplasmin: Macromolecular assemblies with iron-containing acute phase proteins. PLoS ONE 2013, 8, e67145. [CrossRef] [PubMed] 
34. Ashkenazy, H.; Abadi, S.; Martz, E.; Chay, O.; Mayrose, I.; Pupko, T.; Ben-Tal, N. ConSurf 2016: An improved methodology to estimate and visualize evolutionary conservation in macromolecules. Nucleic Acids Res. 2016, 44, W344-W350. [CrossRef] [PubMed]

35. Pettersen, E.F.; Goddard, T.D.; Huang, C.C.; Couch, G.S.; Greenblatt, D.M.; Meng, E.C.; Ferrin, T.E. UCSF Chimera-A visualization system for exploratory research and analysis. J. Comput. Chem. 2004, 25, 1605-1612. [CrossRef] [PubMed]

36. Reese, M.G.; Eeckman, F.H.; Kulp, D.; Haussler, D. Improved splice site detection in Genie. J. Comput. Biol. 1997, 4, 311-323. [CrossRef]

37. Desmet, F.O.; Hamroun, D.; Lalande, M.; Collod-Béroud, G.; Claustres, M.; Béroud, C. Human Splicing Finder: An online bioinformatics tool to predict splicing signals. Nucleic Acids Res. 2009, 37, e67. [CrossRef]

38. Pelucchi, S.; Mariani, R.; Ravasi, G.; Pelloni, I.; Marano, M.; Tremolizzo, L.; Alessio, M.; Piperno, A. Phenotypic heterogeneity in seven Italian cases of aceruloplasminemia. Parkinsonism Relat. Disord. 2018, 51, 36-42. [CrossRef]

39. Miyajima, H.; Takahashi, Y.; Kono, S. Aceruloplasminemia, an inherited disorder of iron metabolism. Biometals 2003, 16, 205-213. [CrossRef]

40. Brissot, P.; Bernard, D.G.; Brissot, E.; Loréal, O.; Troadec, M.B. Rare anemias due to genetic iron metabolism defects. Mutat. Res. 2018, 777, 52-63. [CrossRef]

41. Camaschella, C. How I manage patients with atypical microcytic anemia. Br. J. Haematol. 2013, 160, 12-24. [CrossRef] [PubMed]

42. Donker, A.E.; Raymakers, R.A.; Vlasveld, L.T.; van Barneveld, T.; Terink, R.; Dors, N.; Brons, P.P.; Knoers, N.V.; Swinkels, D.W. Practice guidelines for the diagnosis and management of microcytic anemias due to genetic disorders of iron metabolism or heme synthesis. Blood 2014, 123, 3873-3886. [CrossRef] [PubMed]

43. Vroegindeweij, L.H.P.; Langendonk, J.G.; Langeveld, M.; Hoogendoorn, M.; Kievit, A.J.; Di Raimondo, D.; Wilson, J.H.P.; Boon, A.J.W. New insights in the neurological phenotype of aceruloplasminemia in Caucasian patients. Parkinsonism Relat. Disord. 2017, 36, 33-40. [CrossRef] [PubMed]

44. Miyajima, H.; Hosoi, Y. Aceruloplasminemia. In GeneReviews((R)); Adam, M.P., Ardinger, H.H., Pagon, R.A., Wallace, S.E., Bean, L.J.H., Stephens, K., Amemiya, A., Eds.; University of Washington: Seattle, WA, USA, 1993.

(C) 2020 by the authors. Licensee MDPI, Basel, Switzerland. This article is an open access article distributed under the terms and conditions of the Creative Commons Attribution (CC BY) license (http://creativecommons.org/licenses/by/4.0/). 